\title{
Over 15 years: the advancement of transcatheter mitral valve repair
}

\author{
Paolo Denti, Alessandra Sala, Igor Belluschi, Ottavio Alfieri \\ Department of Cardiovascular and Thoracic Surgery, San Raffaele University Hospital, Milan, Italy \\ Correspondence to: Paolo Denti, MD. Department of Cardiovascular and Thoracic Surgery, San Raffaele University Hospital, Via Olgettina 60, 20132, \\ Milan, Italy. Email: denti.paolo@hsr.it.
}

\begin{abstract}
Patients with severe symptomatic mitral regurgitation, if left untreated, have a poor prognosis. In those patients not eligible for mitral valve (MV) surgery, percutaneous repair may improve clinical outcomes. In the past 15 years several devices have been developed to address different MV lesions. This manuscript will review the advancement of transcatheter MV repair through the years, focusing on technologies for which consistent clinical data is available.
\end{abstract}

Keywords: Mitral regurgitation; mitral valve (MV); MV repair; transcatheter MV repair

Submitted Apr 22, 2020. Accepted for publication Oct 20, 2020.

doi: 10.21037/acs-2020-mv-18

View this article at: http://dx.doi.org/10.21037/acs-2020-mv-18

\section{Introduction}

Mitral valve regurgitation (MR) is the second most common valvular pathology in patients with cardiac valve disease, secondary only to aortic valve stenosis, with a reported prevalence of more than $10 \%$ in people older than 75 years of age $(1,2)$. Severe MR, if left untreated, is associated with progressive left ventricular (LV) dysfunction and congestive heart failure, leading to a high degree of morbidity and mortality $(3,4)$.

Considering the differences in prognosis, indications for treatment and management, it is important to distinguish primary mitral regurgitation (PMR) from secondary mitral regurgitation (SMR). Primary, or organic, $M R$ is characterized by abnormalities in the mitral valve (MV) apparatus, as seen in degenerative disease, rheumatic disease, infective endocarditis and toxic valvulopathy. On the contrary, secondary, or functional MR, is characterized by preserved valvular morphology, in presence of $\mathrm{LV}$ remodeling, displacement of papillary muscles, leaflet tethering and annular dilatation (5-8).

Surgery remains the mainstay of treatment in case of PMR (9); on the contrary it does not play a leading role in SMR, leaving an important window for transcatheter treatments $(10,11)$.
Current European guidelines (12) recommend surgical treatment of severe symptomatic PMR (Class I). Surgery is also indicated in the case of asymptomatic individuals with PMR and other predictors of worse outcome, such as atrial fibrillation and LV dysfunction [defined as ejection fraction (EF) $\leq 60 \%$ and/or LV end systolic diameter (ESD) $\geq 45 \mathrm{~mm}$. For MR, surgery is indicated in patients with severe MR undergoing revascularization with evidence of myocardial viability.

Regardless of the etiology and type of lesions, left untreated severe MR has a poor prognosis, worse quality of life and increase in heart failure symptoms (3). However, in patients with severely reduced LVEF, surgical MV repair or replacement does not lower the rate of hospitalizations and death, and is associated with high surgical risk. Furthermore, it has been estimated that approximately $50 \%$ of patients with severe MR are not eligible for a surgical approach due to age, comorbidities and surgical risk (13). This large unmet clinical need for less-invasive therapeutic options led to the development of transcatheter approaches. These patients may benefit from such procedures, in order to improve symptomatology, quality of life and functional class.

Indeed, since the advent of transcatheter treatment 


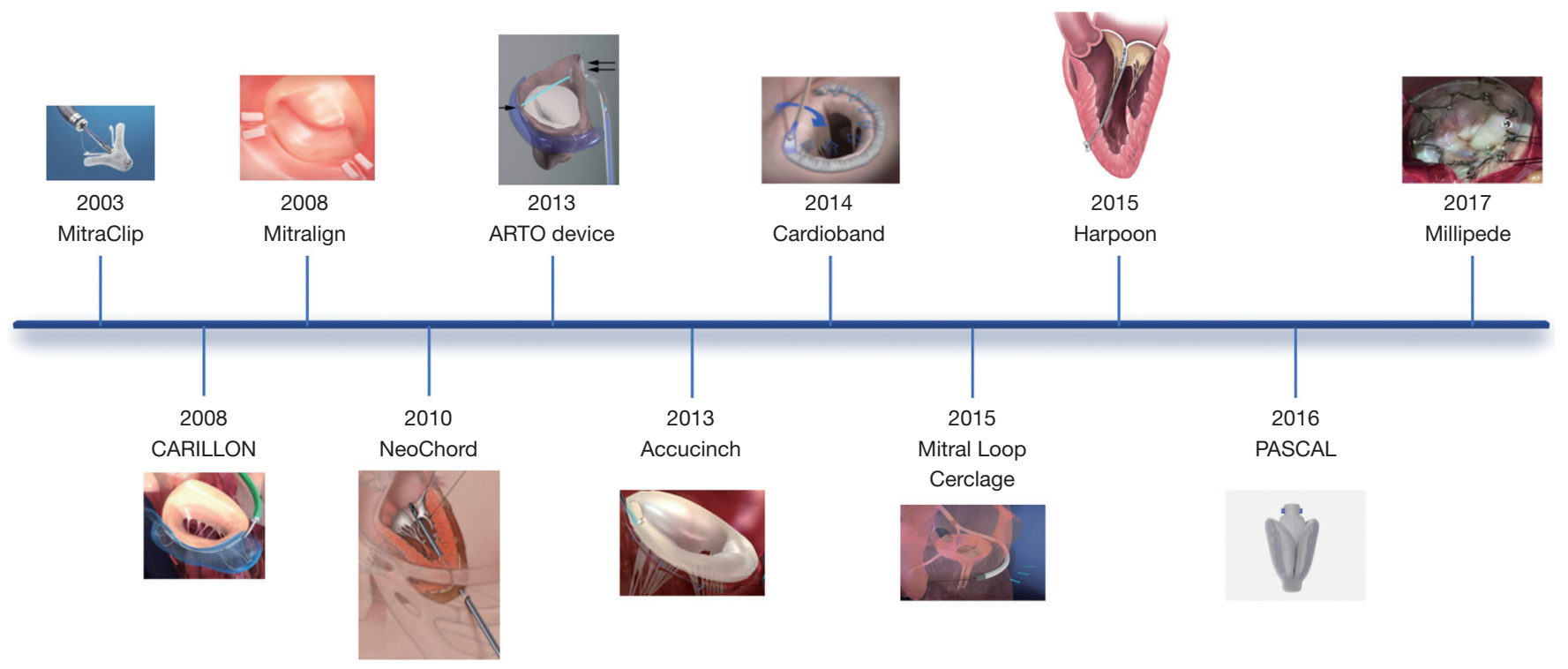

Figure 1 Timeline of the evolving technologies for transcatheter treatment of mitral valve regurgitation.

of aortic valve pathology, great interest has grown in the development of devices capable of addressing MV pathologies. In the past 15 years, technological improvements in percutaneous $\mathrm{MV}$ interventions have made such procedures feasible and safe, and nowadays frequently used in daily clinical practice (14).

In the current review we aim at analyzing the advancements of transcatheter MV repair within the past 15 years, focusing on technologies for which consistent clinical data is available. Particular attention is devoted to achieve a tailored approach choosing the right device according to the specific lesion.

\section{Transcatheter treatment of MR: a selective approach}

Transcatheter MV repair is one of the greatest evolving fields in valvular heart interventions, with a continuous growing number of devices (15).

Different technologies target the great complexity of the MV and the heterogeneous mechanisms of regurgitation (16) (Figure 1). All devices currently available or under development address the various components permitting a tailored approach, mimicking in to the surgical MV repair, as single or possibly combined approaches. The majority of currently available devices can therefore be conveniently categorized into leaflet repair, mitral annuloplasty and chordal implantation (14).

\section{Leaflet repair}

\section{MitraClip device}

The MitraClip system (Abbott Vascular, Abbott Park, IL, USA) replicates the surgical edge-to-edge technique for MV repair, introduced by Alfieri in the surgical armamentarium in 1991 (17). The first-in-human implant occurred in 2003 (18) and subsequently achieved CE mark approval in 2008 and FDA approval in 2013. The device is approved in Europe for treatment of PMR and SMR. Nowadays, more than 100,000 patients have been treated with the MitraClip. Current European Guidelines (12) consider MitraClip treatment as a therapeutic option in high surgical risk patients who meet anatomical criteria of suitability (Class IIb). The procedure is performed with a femoral vein access, and the trans-septal puncture is performed by a steerable guide catheter. The entire procedure is conducted under fluoroscopic and transesophageal echocardiographic guidance. The delivery system is then introduced in the left atrium and the MitraClip device is aligned perpendicularly to the coaptation line of the two MV leaflets. The device is advanced within the left ventricle and upon pull-back, the free edges of the MV leaflets are grasped (19).

The device is composed of two cobalt-chromium arms; the first version of the device, MitraClip NT, has arms of $17 \mathrm{~mm}$ in length and was created for deeper leaflet insertion (Figure 2A). The further version of the device, MitraClip XTR, was modified to $22 \mathrm{~mm}$ in length and 


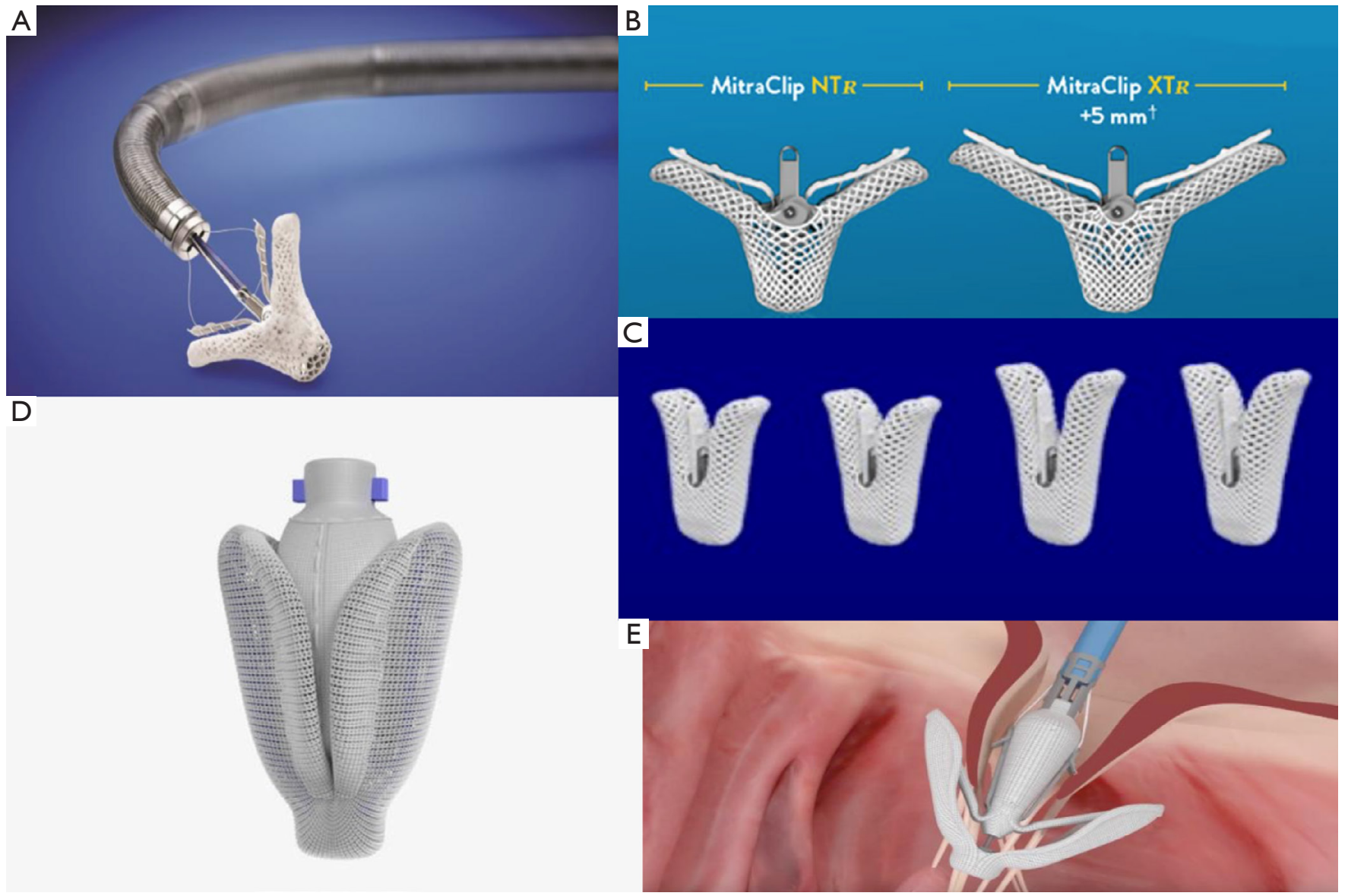

Figure 2 Transcatheter mitral valve repair devices addressing leaflet pathology. (A) MitraClip device; (B) difference between NTR and XTR generations; (C) newer $4^{\text {th }}$ generation MitraClip, available in four sizes; (D) PASCAL device; (E) PASCAL device with two larger paddles and central spacer.

with nitinol gripper lines that enable deeper gripper drop and grasping angle (Figure $2 B$ ). The idea behind increasing the length of the clip was to grip more valvular tissue and possibly expand treatment to more unfavorable anatomies. However, grasping more tissue can convey greater tension on the leaflets causing damage. Furthermore, the longer the clip arms the higher the risk of entrapment within the subvalvular apparatus, especially when addressing commissural lesions. The latest innovation consists of the fourth generation MitraClip device (Figure 2C), available in four different clip sizes, with wider arms, controlled grasping and continuous left atrial pressure monitoring. These changes further allow a tailored repair to each patient's anatomy, allowing treatment of a wider range of challenging anatomies.

Several randomized controlled trials have been conducted with the aim of assessing effectiveness and safety of the MitraClip device. The EVEREST II trial (20) (Endovascular Valve Edge-to-edge Repair Study) was the first multicenter randomized clinical trial that studied 279 patients with MR, comparing percutaneous treatment vs. surgical MV repair or replacement. Results showed, at five years, increased safety of the percutaneous approach (15.0\% major adverse events in MitraClip group vs. 48.0\% in surgical group), and no difference in mortality (18.8\% vs. $21.0 \%)$. Furthermore, a stable MR reduction and positive LV remodeling following MitraClip implant were seen. However, effectiveness resulted to be higher in the surgical group ( $44.2 \%$ vs. $64.3 \%$; $\mathrm{P}=0.01$ ) for higher recurrence of $M R \geq 2+$ and higher reinterventions in the MitraClip group.

Numerous other MitraClip registries have demonstrated a high rate of procedural success and favorable short-term 


\begin{tabular}{|c|c|c|c|c|c|c|c|c|c|}
\hline TRAMI & 1,064 & 75 & $10 \%$ & $87 \%$ & NA & $71 \%$ & $96 \%$ & $5.7 \%$ & NA \\
\hline European sentinel & 628 & 74 & $20 \%$ & $86 \%$ & $43 \%$ & $72 \%$ & $98 \%$ & $3 \%$ & $15.3 \%$ \\
\hline ACCESS-EU & 567 & 78 & $23 \%$ & $85 \%$ & NA & $77 \%$ & $91 \%$ & $3.4 \%$ & $17.3 \%$ \\
\hline GRASP & 171 & 71 & $7 \%$ & $81 \%$ & $37 \%$ & $78 \%$ & $93 \%$ & $0.9 \%$ & $14 \%$ \\
\hline MitraSwiss & 100 & 77 & $17 \%$ & $82 \%$ & $48 \%$ & $62 \%$ & $85 \%$ & $4 \%$ & $15.4 \%$ \\
\hline
\end{tabular}

outcomes (Table 1), and many more are still ongoing $(21,22)$.

Recently, transcatheter treatment of SMR has been under the spotlight due to release of two multicenter randomized studies performed in order to assess effectiveness of MitraClip treatment in patients with heart failure and SMR, that remained symptomatic despite maximal medical therapy. Both randomized patients to treatment with medical therapy alone, or MitraClip together with medical therapy. MITRA-FR (23) study results showed $M R \leq 2$ in $92 \%$ of patients following the procedure and a re-hospitalization rate for heart failure within the first year of follow-up of $55.0 \% \mathrm{vs} .51 .0 \%$ in the interventional and control groups, respectively. At twelve months, the mortality rate was $24.0 \%$ in the former vs. $22.0 \%$ in the latter [hazard ratio (HR), 1.11; 95\% CI, 0.69-1.77]. On the contrary, the COAPT study (24), for the first time ever, strongly demonstrated a lower all-cause mortality and reduced hospitalizations for heart failure events in the device group in comparison the control group. The benefits were consistent throughout subgroups, including ischemic and non-ischemic cardiomyopathy, $\mathrm{LV}$ volume and function and baseline. Possible explanations for such different results were the considerable amount of missing echocardiographic data at twelve months in the French study, patient selection and length of follow-up.

\section{PASCAL system}

The PASCAL device (Edwards Lifesciences, Irvine, CA)from "Paddles, Spacer, Clasps, Alfieri"—was designed to treat MR by overcoming some of the limitations of the previous system. The device has larger paddles with a central spacer in order to maximize leaflet coaptation and the ability to grasp the individual leaflets (Figure 2D). Furthermore, the clasps allow for independent leaflet capture and offer the possibility to finetune leaflet positioning (Figure 2E). Delivery of the device occurs through a transvenous transfemoral access and transseptal approach. The device received CE mark in February 2019, and is still awaiting FDA approval. The multicenter, prospective CLASP trial $(25,26)$ addresses the feasibility of the new Edwards device in patients affected by both PMR and SMR. Mean age was 76.5 years, with the majority of patients having FMR, 51.6\%, vs. 36.0\% with DMR and $8.0 \%$ with a mixed etiology. Results showed a low cardiovascular mortality (1.6\%) without any stroke events. Furthermore, $98 \%$ of patients at follow-up had MR $\leq 2+$, among which $86 \%$ with $M R \leq 1+$, and the great majority of patients resulted in improvement in functional class, with $85 \%$ of them being in NYHA Class I/II.

A new randomized trial, the CLASP IID/F has begun enrolment and will aim at comparing the efficacy and safety of the PASCAL device compared to the MitraClip device in patients with significant PMR and SMR. However, results of the first CLASP trial made various questions emerge regarding the role of the two devices. The PASCAL device represents an important advancement in the treatment of $M R$, and will likely play a converging role together with the MitraClip system. In fact, the MitraClip system has some intrinsic limitations, and it is not suitable for the entire proportion of patients due to anatomical reasons, the socalled EVEREST criteria. Furthermore, the edge-to-edge repair performed with the MitraClip can be demanding in some anatomical settings due to difficult maneuvering of the system (Table 2). The novel leaflet repair device was designed in order to overcome such limitations, trying to expand patient eligibility for repair (27). Additional data on durability and complementary role with the MitraClip device needs to be further investigated. 
Table 2 Anatomical considerations for MitraClip treatment
(EVEREST Criteria)

Favorable
Moderate-severe pr severe MR
A2-P2 defect
Prolapse width $<15 \mathrm{~mm}$
Flail gap $<10 \mathrm{~mm}$
Mitral valve orifice area $>4 \mathrm{~cm}^{2}$
Mobile length posterior leaflet $\geq 7 \mathrm{~mm}$
Unfavorable/challenging
Commissural lesions
Clefts
Short posterior leaflet $(<5 \mathrm{~mm})$
Mitral valve orifice area $<3.5 \mathrm{~cm}^{2}$
Severe calcification of grasping zone
Leaflet perforations
Mitral stenosis Gmed $\geq 5 \mathrm{mmHg}$ OR active endocarditis

\section{Mitral annuloplasty}

Reduction of the MV annulus with an undersized ring is the standard surgical treatment for functional MR. Only with annuloplasty it is possible to restore MV competency, improve LV performance, obtain reverse remodeling and decrease symptoms.

Development of less invasive, percutaneous devices, has evolved over the years as a valuable alternative to surgical annuloplasty, especially in high-risk patients. In fact, up to one-third of patients screened for MitraClip are declined leaflet repair due to unfavorable anatomy, including marked annular dilation. The unmet clinical need together with the will to address ineligible patients drove the development of a great variety of devices. Placing greater emphasis on the devices currently available, these can be further subdivided into two groups: direct and indirect annuloplasty systems.

\section{Indirect annuloplasty devices}

\section{CARILLON mitral contour system}

The CARILLON device (Cardiac Dimensions, Inc., Kirkland, Washington) was the principal system to be investigated in this subcategory of devices and received CE mark approval in 2011. It consists of a self-expandable nitinol semi-helical distal and proximal anchors connected by a nitinol bridge and delivered through a transjugular venous access (Figure $3 A, B$ ). The proximal anchor is positioned at the level of the coronary sinus, while the distal anchor within the great cardiac vein. Upon release the system is capable of plicating the posterior MV annulus to reduce annular dilation and, as a consequence, the degree of MR. The device is therefore indicated in patients with functional MR, and can be easily retrieved and repositioned if MR reduction is not adequate or if coronary artery compromise is detected (28).

Initial studies of the CARILLON device, AMADEUS (28) and TITAN (29) trials have shown good results of this annuloplasty system. The AMADEUS trial, a prospective single-arm feasibility study, showed significant reduction in mitral annulus diameter, decrease in degree of MR by at least one grade, improvement in functional class and quality of life at 24 months follow-up. The second trial performed, the TITAN trial (The Transcatheter Implantation of the Carillon Mitral Annuloplasty Device), was a prospective non-randomized study of patients with functional MR treated with a second-generation device. Fifty-three patients were enrolled, and results reported successful permanent device implantation in 36 patients. Furthermore, there was evidence of significant reduction in degree of $M R$, including regurgitant volume and effective regurgitant orifice area (EROA). Positive LV remodelling was demonstrated at 12 months, together with improvement in functional class and quality of life at 24 months. In seventeen of the 53 patients, the device could not be permanently implanted due to difficulty cannulating the coronary sinus, ineffective reduction in $\mathrm{MR}$, or compression of the circumflex artery. The TITAN II study (30) was conducted in order to test the newer generation of the modified CARILLON device. A single device fracture was reported, and one year mortality was $23 \%$, with no death adjudicated to be device-related. The modified CARILLON device is currently being evaluated in a multicenter blinded randomized control trial, the REDUCE FMR trial.

Due to the favorable position of the coronary sinus, it is noteworthy to mention other indirect annuloplasty devices developed in the past that took advantage of the same mechanism of action. These include the Monarc device (31) (Edwards Lifesciences, Irvine, California) that was studied in a human trial, but was subsequently halted due to slow enrollment, and the Viacor PTMA system (32) (Viacor, Wilmington, Massachusetts) that was taken off the market due to late, fatal coronary sinus laceration. In 


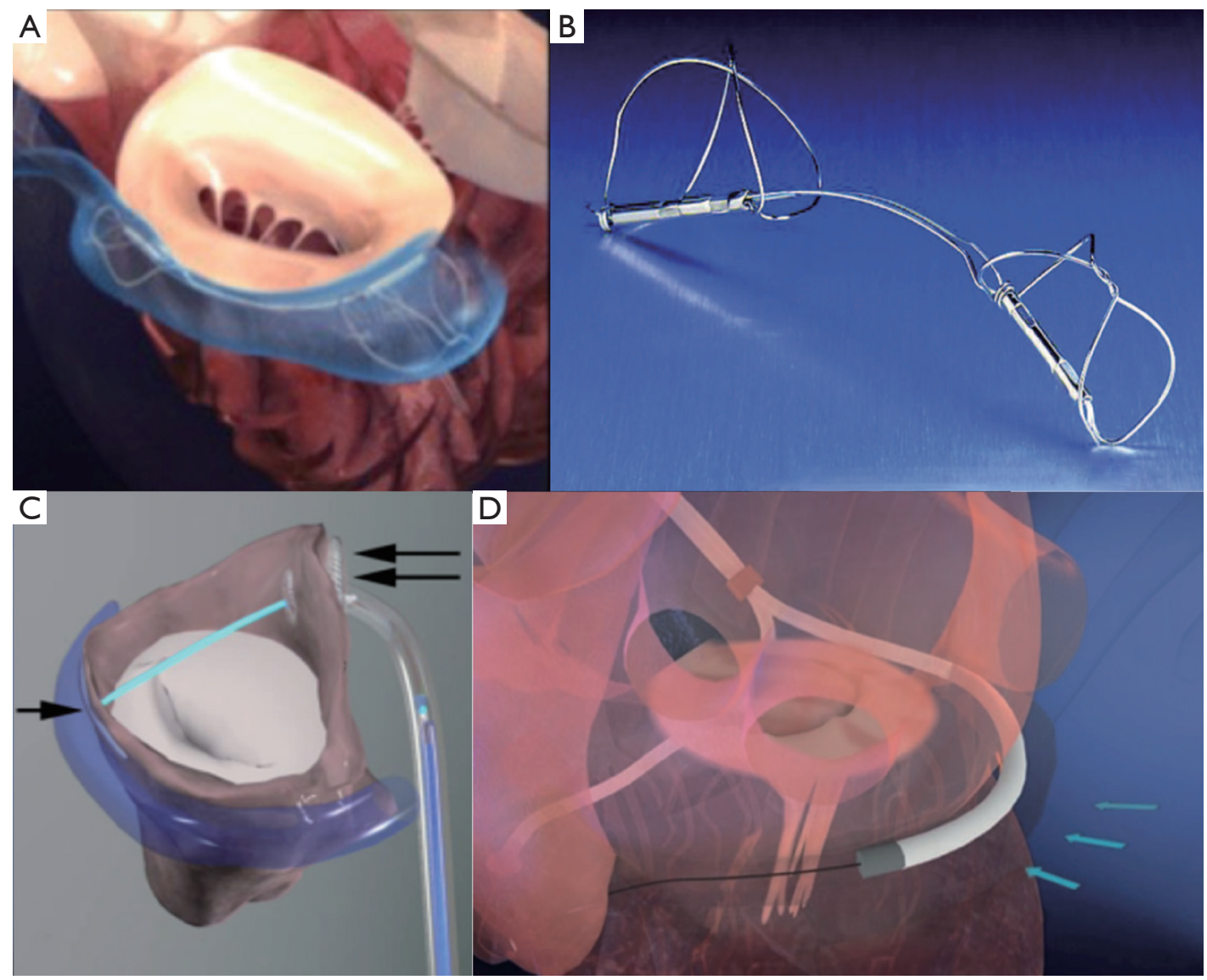

Figure 3 Indirect annuloplasty devices. (A,B) CARILLON Mitral Contour System; (C) MVRx ARTO (arrows); (D) Mitral Loop Cerclage (arrows).

fact, regardless the "safe" position of the coronary sinus, non-invasive imaging has demonstrated that the separation between the coronary sinus and the mitral apparatus increases significantly in dilated hearts compared to normal hearts, resulting in ineffective MR reduction. This may require increased cinching with a higher probability of compressing the circumflex artery or major branches.

\section{ARTO device}

The ARTO device: the MVRx ARTO transcatheter annular reduction therapy (MVRX, Inc., Belmont, California) is a system that delivers transvenously two anchors: one through the interatrial septum, the second to the coronary sinus, and acts by reducing the antero-posterior diameter of the mitral annulus (Figure 3C). The MV RepaIr Clinical trial (MAVERIC trial) (33) is a prospective single arm study that evaluated safety and performance of the device in patients affected by functional MR. Promising results of the first patients treated (11/31) have been released, but complete and final results are still pending.

\section{Mitral loop cerclage catheter system}

The Mitral Loop Cerclage system (34) (Tau-PNU Medical Co, Ltd., Pusan, Korea) is a procedure performed with a double venous access: a femoral vein access and a left subclavian vein one, via a pacemaker-type pocket (Figure 3D). The cerclage is accomplished using a guidewire to enter the coronary sinus and great cardiac vein, and snaring of the wire occurs from the right ventricular outflow tract once crossing of the interventricular septum across the anterior interventricular vein is obtained. A loop is formed around the mitral annular plane and the guidewire is exchanged for a tension device that allows compression of the mitral annulus improving leaflet coaptation. The device contains an integrated coronary artery protection element. The tension locking device is then embedded within the left 
sub-clavicular pocket.

The first-in-human study (35) was performed on five patients, with successful implantation in four individuals. In one patient the procedure was aborted due to unfavorable anatomy. Implant of the device resulted in immediate reduction of MR grade, which was sustained at six months, together with reduction in left atrial and ventricular chamber volumes. Of note, the major device-related complications were coronary artery occlusion and new bundle branch block.

Even though constant improvements and inventions are being made in the field of indirect annuloplasty for the treatment of functional MR, limited application of such devices is strongly dependent on anatomic variations of both the coronary sinus and coronary anatomy as a whole.

\section{Direct annuloplasty devices}

Direct annuloplasty devices more closely reproduce surgical annuloplasty techniques (Figure 4). The posterior annulus is usually only targeted for this purpose, since the anterior MV annulus remains a more challenging structure to target, with its anatomical proximity to the aortic valve.

\section{Cardioband device}

The Cardioband system (36) (Valtech Cardio Ltd., Or Yehuda, Israel) is a device delivered via a transvenous transseptal approach, which received CE mark approval in 2015. The mechanism consists in delivering direct sutureless helical anchors on the atrial side of the mitral annulus in order to connect the annuloplasty polyester prosthetic tube, from the antero-lateral to the posteromedial trigone. Following implantation, the diameter of the annulus is reduced by controlling tension on the band, under echocardiographic guidance.

Recent results have been published regarding the largest multicenter study, comprising 60 patients affected by functional MR who underwent Cardioband implantation (37). Early results documented anchor detachment, therefore device modification occurred half-way through the study with net improvement (nine of ten patients occurred prior to device modification). Survival at one year was $87 \%$ and no device-related deaths were reported. Furthermore, at one year, MR grade was moderate or less in $61 \%$ of patients treated, with a significant improvement in functional class, quality of life and exercise capacity.

Of striking interest is the ongoing pivotal ACTIVE Trial that is designed to demonstrate the superiority of
Cardioband and guidelines-directed medical therapy (GDMT) compared to GDMT alone, in patients with clinically significant SMR, with a composite hierarchical endpoint of cardiovascular mortality, heart failure hospitalization, quality of life, and exercise capacity.

It is noteworthy to underline that the Cardioband device has the great advantage of preserving the patient's native anatomy, therefore allowing to keep future repair options open. Furthermore, when considering treatment with multiple device therapies, the Cardioband device has been combined with both double orifice repair with the MitraClip (38) device and chordal replacement in order to maximize MR reduction. Still, several studies have to be made in order to better define the potential future of such combination.

\section{Mitralign annuloplasty system}

The Mitralign Percutaneous Annuloplasty device (Mitralign, Tewksbury, MA, USA) is a transfemoral arterial access deflectable catheter that aims at plicating the posterior mitral annulus. The device received CE mark approval in 2016. The procedure is performed by advancing a catheter retrogradely into the left ventricle and guidewires penetrate the annulus into the left atrium, where pledgets are implanted at the level of P1-P2 and P2$\mathrm{P} 3$. Each pledget pair can be pulled together resulting in a segmental annuloplasty capable of shortening the annulus. A prospective, multicenter single-arm feasibility study (ALIGN Trial) was performed on 71 patients, among which 50 underwent Mitralign implant (39). No intraprocedural death was reported, however $8 \%$ experienced pericardial tamponade, and at six months follow-up all-cause mortality was $12.2 \%$, with seven patients requiring MitraClip implantation and one patient receiving non-emergent $\mathrm{MV}$ surgery, therefore the device is no longer available on the market. MR improvement was seen in $50 \%$ of patients treated, with a higher trend in patients that received two pledgets.

\section{AccuCinch ventricular repair system}

The AccuCinch ventricular repair device (40) (Ancora Heart, Santa Clara, CA) uses a retrograde approach via a transfemoral arterial access in order to implant a series of adjustable anchors within the LV wall. A cable is found below the MV annulus and it can be tightened to cinch the LV wall. This causes reduction in LV size, and as a consequence reduction in MV annulus. Overall, it can be considered a ventriculoplasty rather than a direct 


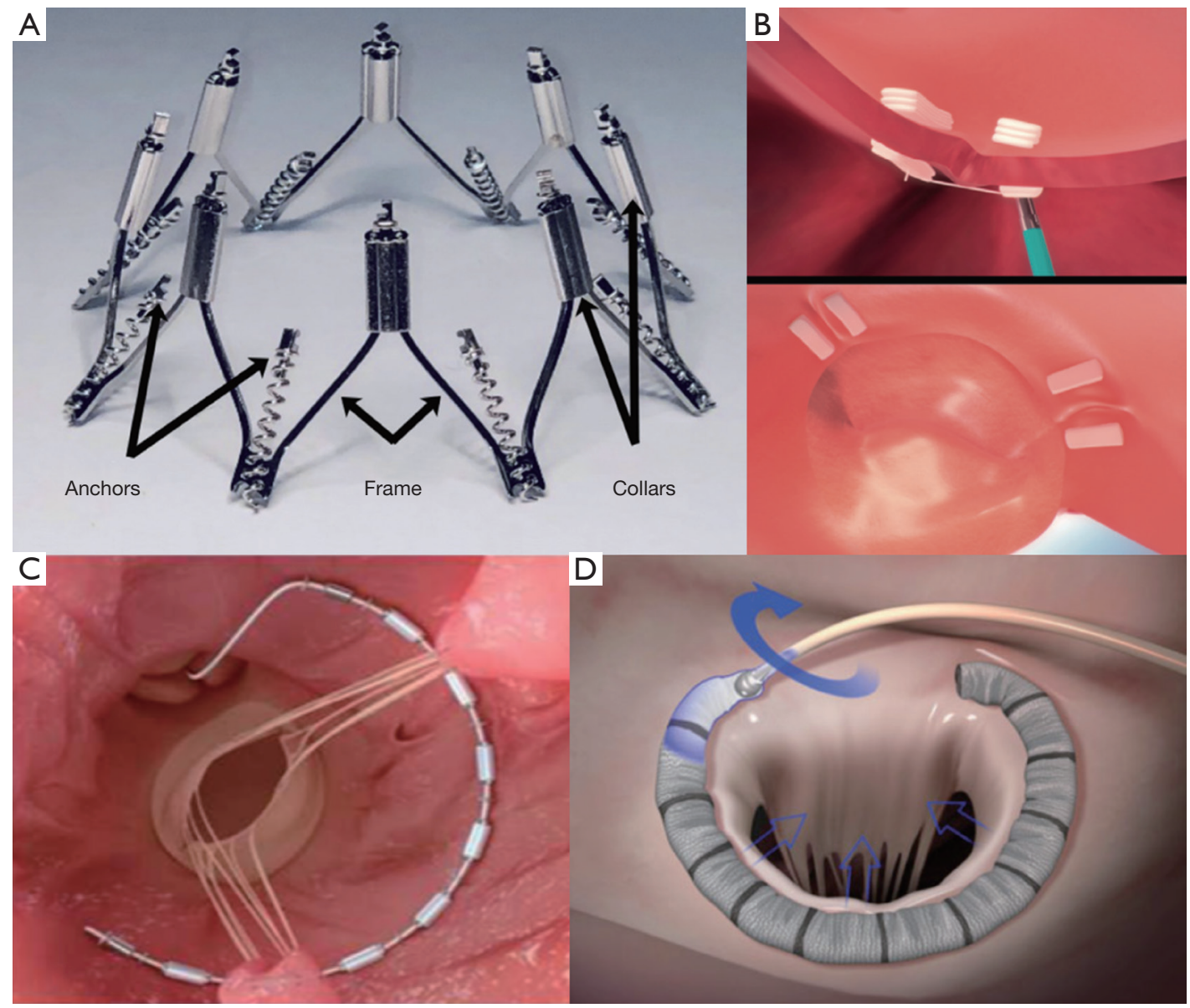

Figure 4 Direct annuloplasty devices. (A) Millipede IRIS Ring; (B) Mitralign annuloplasty system; (C) AccuCinch Ventricular Repair device; (D) Cardioband device.

annuloplasty device, and it is in fact also being tested in patients with heart failure and without significant valvular lesions. Prospective data on this device is still missing. However, two studies, LVRECOVER and LVRESTORESA, involving around ten patients were performed, both with promising results.

\section{Millipede IRIS ring}

The Millipede IRIS Ring device (Millipede, Inc., Santa Rosa, CA) is a semi-rigid complete ring that is delivered through a transfemoral transseptal approach. It consists of eight helical stainless-steel anchors pre-attached to the base of the device. Each anchor rotates independently and attaches directly to the mitral annulus. Each anchor can also be retracted or unscrewed if necessary. When tensioned, adjacent anchors are brought closer together, and the crowns can be tightened individually in order to selectively reduce the most dilated portions of the MV annulus. Results on the very few first patients treated seem promising, with good reduction in MV annulus diameter (41). However, more detailed procedural and follow-up results will be further evaluated in ongoing clinical trials (42).

\section{Chordal replacement}

Among the different surgical repair techniques and strategies, a valuable alternative in patients affected by PMR is the so-called "respect rather than resect", with the implantation of one or more artificial chordae. The development of less-invasive transapical chordal replacement has seen great development worldwide.

\section{NeoChord DS1000 System}

The NeoChord DS1000 device (43) (Neochord, Inc., 

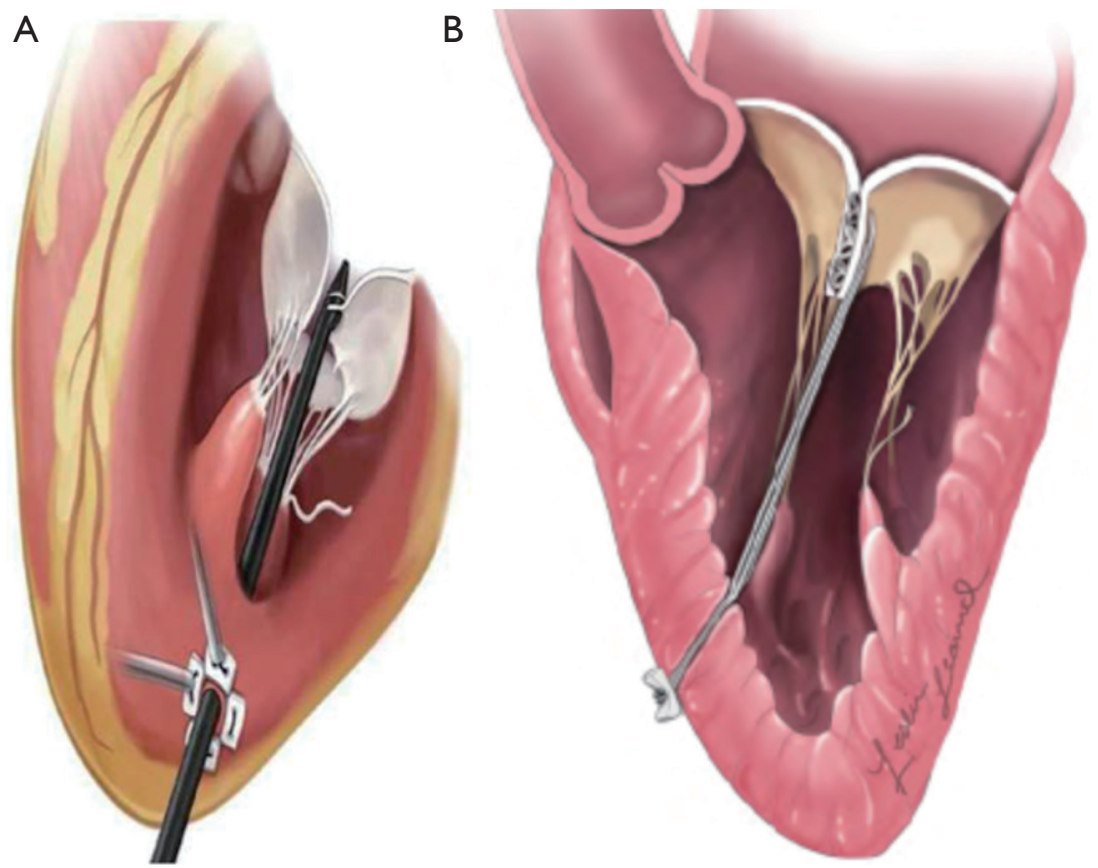

Figure 5 Chordal Replacement devices. (A) NeoChord System; (B) HARPOON Medical system.

St. Louis Park, MN, USA) was the first device designed to allow transapical beating heart implantation of PTFE chordal loops on the MV leaflet to correct flail or prolapse, and obtained CE mark approval in 2012 Figure 5A. The initial TACT trial (44) demonstrated acute procedural success in 26 of 30 patients, with only 17 patients with MR grade $<2+$ at 30 days. Recently, one year follow-up of the largest International Registry comprising 213 patients was published. Procedural success was achieved in $96.7 \%$ of patients, with an overall survival at one year of $98 \% \pm 1 \%$. Composite endpoint, defined as procedural success, freedom from mortality, stroke, reintervention, recurrence of severe MR, rehospitalization and decreased of $\geq$ NYHA class at follow-up, was achieved in $84 \% \pm 2.5 \%$ of the overall population $(45,46)$. Furthermore, a clinical trial, ReChord, is a randomized trial currently enrolling patients comparing NeoChord transapical system $v s$. open surgical mitral repair in patients with degenerative MR.

While experience continues to increase, a critical drawback worth mentioning is the inability of such devices to restore even coaptation along the entire flailing/ prolapsing segment. In fact, chordal loops provide focal support to the leaflet, and inter-loop prolapse and regurgitation can persist after the repair. This, together with a study conducted by Weber et al. (47), in which attachment of chordae to ventricular apex seemed to increase the risk of systolic anterior motion, may explain the relative high-rate of repair failure in some implants.

\section{HARPOON Device}

The HARPOON Medical System (48) (Edwards Lifesciences Corporation, Irvine, CA) is a more recent addition to this category of transcatheter repair techniques (Figure 5B). The procedure is performed through a small left thoracotomy. The device consists of a hemostatic $14 \mathrm{Fr}$ introducer and a delivery system that contains a pre-formed PTFE knot that anchors chords on target locations on the MV leaflet. After each knot deployment the device is withdrawn, exteriorizing two PTFE chordae. The number of knots deployed greatly depends on the extent of the flailing/prolapsing segment. The suture pairs are then threaded through a pledget and tightened under echocardiographic guidance to optimize coaptation and reduce MR grade. The device is being evaluated through a prospective, non-randomized, multicenter clinical study, the TRACER trial, in order to assess safety and performance. Thirty patients have been treated with severe PMR. Three patents required conversion to MV surgery. Twentyseven of thirty $(90 \%)$ patients reached primary endpoint of successful implantation and reduction of MR grade to 
moderate-or-less. At six months, MR was moderate or less in $85 \%$ of patients, moderate in $8 \%$ and severe in the remaining $8 \%$. Favorable cardiac remodeling was seen as a decrease in LV end-diastolic volume and left atrial volumes. Longer follow-up, together with further investigations are required. However, results seem promising, and may be linked to the preformed knot and the support to a larger section of the leaflet, as compared to the NeoChord device.

\section{Pipeline}

Pipeline device (Gore Medical, USA) is another transcatheter device designed for off-pump beating heart chordal replacement. The device is delivered via a transfemoral transseptal access; a guidewire is driven across the MV, within the left ventricle. Pipeline device is advanced to the papillary muscles and the distal ventricular or papillary muscle helical anchor in deployed. The following step consists in puncturing the MV leaflet in order to obtain leaflet fixation. Suture length adjustment is obtained by means of a suture lock device, under transesophageal echocardiography guidance. The artificial Neochord is tensioned in order to obtain the best coaptation and optimal MR reduction.

\section{Repair vs. replacement}

Despite increasing evidence of feasibility and good outcome with transcatheter repair options, almost $25 \%$ of treated patients will face at least moderate residual MR (49). Therefore, improvements in technology and in learning curves may play a pivotal role in the next years. As for surgery, whenever MV repair is possible, it should be considered as first choice treatment. In fact, several factors justify the adoption of a "repair-first strategy", even in the percutaneous context, including: low early mortality rates, the absence of valve-related events (such as structural valve deterioration), a more respectful approach towards leaflet and annulus, higher suitability, and a shorter screening process (50). However, if a good MV repair is not expected, transcatheter MV replacement should be considered.

\section{Future directions}

Looking towards the future and also at the next European Society of Cardiology guidelines for the treatment of MR, it is possible to state that less invasive mitral procedures are gaining their field of interest. With the great development of technologies, together with the very important creation of Heart Teams, the various transcatheter devices need to be selected and utilized according to valve anatomy, etiology, valvular lesion and general condition of the patient, in order to offer the best possible treatment to every individual and gain a real patient-tailored approach. In fact, the Heart Team concept will further improve the decision-making process on "which device to which patient". Considering PMR, surgery remains the treatment of choice, to date, however transcatheter interventions will likely provide a palliation in patients considered very highrisk or inoperable. On the contrary, in SMR a wider role can be further expected in the future for transcatheter repair devices (51).

A further step in the future will be to systematically combine repair techniques, such as leaflet repair or chordal replacement together with percutaneous annuloplasty, aiming at reproducing surgical long-term durable results. Furthermore, in the next few years we will see results of many trials that are currently on their way and this will hopefully help us in identifying an even clearer therapeutic line to follow.

\section{Conclusions}

In this manuscript we analyzed the principal devices that in the last 15 years have been invented, developed and further improved in order to provide safe and effective repair options for the treatment of MR. Some of these devices have paved the way for the growing importance of transcatheter MV repair as a novel therapeutic alternative. Such treatments have come a long way, with several trials completed and still a great number ongoing. We reviewed the main devices currently available, and their possible use according to etiology of MR and valvular lesion.

\section{Acknowledgments}

Funding: None.

\section{Footnote}

Conflicts of Interest: The authors have no conflicts of interest to declare.

Open Access Statement: This is an Open Access article distributed in accordance with the Creative Commons 
Attribution-NonCommercial-NoDerivs 4.0 International License (CC BY-NC-ND 4.0), which permits the noncommercial replication and distribution of the article with the strict proviso that no changes or edits are made and the original work is properly cited (including links to both the formal publication through the relevant DOI and the license). See: https://creativecommons.org/licenses/by-nc-nd/4.0/.

\section{References}

1. Nkomo VT, Gardin JM, Skelton TN, et al. Burden of valvular heart diseases: a population-based study. Lancet 2006;368:1005-11.

2. Iung B, Baron G, Butchart EG, et al. A prospective survey of patients with valvular heart disease in Europe: The Euro Heart Survey on valvular heart disease. Eur Heart J 2003;24:1231-43.

3. Goel SS, Bajaj N, Aggarwal B, et al. Prevalence and outcomes of unoperated patients with severe symptomatic mitral regurgitation and heart failure: Comprehensive analysis to determine the potential role of mitraclip for this unmet need. J Am Coll Cardiol 2014;63:185-6.

4. Asgar AW, Mack MJ, Stone GW. Secondary mitral regurgitation in heart failure: Pathophysiology, prognosis, and therapeutic considerations. J Am Coll Cardiol 2015;65:1231-48.

5. Nishimura RA, Otto CM, Bonow RO, et al. 2017 AHA/ ACC Focused update of the 2014 AHA/ACC guideline for the management of patients with valvular heart disease. Circulation 2017;135:e1159-95.

6. Otsuji Y, Kumanohoso T, Yoshifuku S, et al. Isolated annular dilation does not usually cause important functional mitral regurgitation: Comparison between patients with lone atrial fibrillation and those with idiopathic or ischemic cardiomyopathy. J Am Coll Cardiol 2002;39:1651-6.

7. Sattur S, Bates S, Movahed MR. Prevalence of mitral valve prolapse and associated valvular regurgitations in healthy teenagers undergoing screening echocardiography. Exp Clin Cardiol 2010;15:e13-5.

8. Kihara T, Gillinov AM, Takasaki K, et al. Mitral regurgitation associated with mitral annular dilation in patients with lone atrial fibrillation: An echocardiographic study. Echocardiography 2009;26:885-9.

9. Enriquez-Sarano M. Timing of mitral valve surgery. Heart 2002;87:79-85.

10. Acker MA, Parides MK, Perrault LP, et al. Mitral-valve repair versus replacement for severe ischemic mitral regurgitation. N Engl J Med 2014;370:23-32.

11. Michler RE, Smith PK, Parides MK, et al. Two-year outcomes of surgical treatment of moderate ischemic mitral regurgitation. N Engl J Med 2016;374:1932-41.

12. Baumgartner H, Falk V, Bax JJ, et al. 2017 ESC/EACTS Guidelines for the management of valvular heart disease. Eur Heart J 2017;38:2739-91.

13. Mirabel M, Iung B, Baron G, et al. What are the characteristics of patients with severe, symptomatic, mitral regurgitation who are denied surgery? Eur Heart J 2007;28:1358-65.

14. Feldman T, Young A. Percutaneous approaches to valve repair for mitral regurgitation. J Am Coll Cardiol 2014;63:2057-68.

15. Nishimura RA, Vahanian A, Eleid M, et al. Mitral valve disease. current management and future challenges. Lancet 2016;387:1324-34.

16. Alfieri O, Buzzatti N. Expansion of the treatment toolbox for mitral regurgitation. Lancet 2017;390:722-4.

17. Alfieri O, Maisano F, De Bonis M, et al. The doubleorifice technique in mitral valve repair: A simple solution for complex problems. J Thorac Cardiovasc Surg 2001;122:674-81.

18. Feldman T, Wasserman HS, Herrmann HC, et al. Percutaneous mitral valve repair using the edge-to-edge technique: Six-month results of the EVEREST phase I clinical trial. J Am Coll Cardiol 2005;46:2134-40.

19. Sherif MA, Paranskaya L, Yuecel S, et al. Mitraclip step by step; how to simplify the procedure. Neth Heart J 2017;25:125-30.

20. Feldman T, Kar S, Elmariah S, et al. Randomized Comparison of Percutaneous Repair and Surgery for Mitral Regurgitation 5-Year Results of EVEREST II. J Am Coll Cardiol 2015;66:2844-54.

21. Puls M, Lubos E, Boekstegers P, et al. One-year outcomes and predictors of mortality after MitraClip therapy in contemporary clinical practice: Results from the German transcatheter mitral valve interventions registry. Eur Heart J 2016;37:703-12.

22. Maisano F, Franzen O, Baldus S, et al. Percutaneous mitral valve interventions in the real world: Early and 1-year results from the ACCESS-EU, A prospective, multicenter, nonrandomized post-approval study of the Mitraclip therapy in Europe. J Am Coll Cardiol 2013;62:1052-61.

23. Obadia JF, Messika-Zeitoun D, Leurent G, et al. Percutaneous repair or medical treatment for secondary 
mitral regurgitation. N Engl J Med 2018;379:2297-306.

24. Stone GW, Lindenfeld JA, Abraham WT, et al.

Transcatheter mitral-valve repair in patients with heart failure. N Engl J Med 2018;379:2307-18.

25. Praz F, Spargias K, Chrissoheris M, et al. Compassionate use of the PASCAL transcatheter mitral valve repair system for patients with severe mitral regurgitation: a multicentre, prospective, observational, first-in-man study. Lancet 2017;390:773-80.

26. Lim DS, Kar S, Spargias K, et al. Transcatheter Valve Repair for Patients With Mitral Regurgitation: 30-Day Results of the CLASP Study. JACC Cardiovasc Interv 2019;12:1369-78.

27. Grasso C, Rubbio AP. The PASCAL transcatheter mitral valve repair system for the treatment of mitral regurgitation: Another piece to the puzzle of edge-to-edge technique. J Thorac Dis 2017;9:4856-9.

28. Schofer J, Siminiak T, Haude M, et al. Percutaneous Mitral Annuloplasty for Functional Mitral Regurgitation. Circulation 2009;120:326-33.

29. Siminiak T, Wu JC, Haude M, et al. Treatment of functional mitral regurgitation by percutaneous annuloplasty: Results of the TITAN Trial. Eur J Heart Fail 2012;14:931-8.

30. Lipiecki J, Siminiak T, Sievert H, et al. Coronary sinusbased percutaneous annuloplasty as treatment for functional mitral regurgitation: The TITAN II trial. Open Heart 2016;3:e000411.

31. Harnek J, Webb JG, Kuck KH, et al. Transcatheter implantation of the MONARC coronary sinus device for mitral regurgitation. JACC Cardiovasc Interv 2011;4:115-22.

32. Sack S, Kahlert P, Bilodeau L, et al. Percutaneous transvenous mitral annuloplasty initial human experience with a novel coronary sinus implant device. Circ Cardiovasc Interv 2009;2:277-84.

33. Rogers JH, Thomas M, Morice MC, et al. Treatment of Heart Failure with Associated Functional Mitral Regurgitation Using the ARTO System Initial Results of the First-in-Human MAVERIC Trial (Mitral Valve Repair Clinical Trial). JACC Cardiovasc Interv 2015;8:1095-104.

34. Kim JH, Sung SC, Chon MK, et al. Mitral loop cerclage as a variant form of mitral cerclage annuloplasty that adds a device (CSTV) for preventing potential complications: A preclinical proof of concept and feasibility study. EuroIntervention 2016;11:e1669-79.

35. Park YH, Chon MK, Lederman RJ, et al. Mitral Loop Cerclage Annuloplasty for Secondary Mitral
Regurgitation: First Human Results. JACC Cardiovasc Interv 2017;10:597-610.

36. Maisano F, Taramasso M, Nickenig G, et al. Cardioband, a transcatheter surgical-like direct mitral valve annuloplasty system: Early results of the feasibility trial. Eur Heart J 2016;37:817-25.

37. Messika-Zeitoun D, Nickenig G, Latib A, et al. Transcatheter mitral valve repair for functional mitral regurgitation using the Cardioband system: 1 year outcomes. Eur Heart J 2019;40:466-72.

38. Latib A, Ancona MB, Ferri L, et al. Percutaneous Direct Annuloplasty With Cardioband to Treat Recurrent Mitral Regurgitation After MitraClip Implantation. JACC Cardiovasc Interv 2016;9:e191-2.

39. Nickenig G, Schueler R, Dager A, et al. Treatment of Chronic Functional Mitral Valve Regurgitation With a Percutaneous Annuloplasty System. J Am Coll Cardiol 2016;67:2927-36.

40. Gooley RP, Meredith IT. The Accucinch transcatheter direct mitral valve annuloplasty system. EuroIntervention 2015;11 Suppl W:W60-1.

41. Rogers JH, Boyd WD, Smith TW, et al. Early experience with Millipede IRIS transcatheter mitral annuloplasty. Ann Cardiothorac Surg 2018;7:780-6.

42. Rogers JH, Boyd WD, Smith TW, et al. Transcatheter Mitral Valve Direct Annuloplasty with the Millipede IRIS Ring. Interv Cardiol Clin 2019;8:261-7.

43. Seeburger J, Borger MA, Tschernich H, et al. Transapical beating heart mitral valve repair. Circ Cardiovasc Interv 2010;3:611-2.

44. Seeburger J, Rinaldi M, Nielsen SL, et al. Off-pump transapical implantation of artificial neo-chordae to correct mitral regurgitation: The tact trial (transapical artificial chordae tendinae) proof of concept. J Am Coll Cardiol 2014;63:914-9.

45. Colli A, Adams D, Fiocco A, et al. Transapical NeoChord mitral valve repair. Ann Cardiothorac Surg 2018;7:812-20.

46. Colli A, Manzan E, Aidietis A, et al. An early European experience with transapical off-pump mitral valve repair with NeoChord implantation. Eur J Cardiothorac Surg 2018;54:460-6.

47. Savic V, Pozzoli A, Gülme G, et al. Transcatheter mitral valve chord repair. Ann Cardiothorac Surg 2018;7:731-40.

48. Gammie JS, Wilson P, Bartus K, et al. Transapical beating-heart mitral valve repair with an expanded polytetrafluoroethylene cordal implantation device. Circulation 2016;134:189-97.

49. Nickenig G, Estevez-Loureiro R, Franzen O, et al. 
Percutaneous mitral valve edge-to-edge Repair: In-hospital results and 1-year follow-up of 628 patients of the 20112012 pilot European Sentinel Registry. J Am Coll Cardiol 2014;64:875-84.

50. Taramasso M, Gavazzoni M, Nickenig G, et al.

Transcatheter mitral repair and replacement: which

Cite this article as: Denti P, Sala A, Belluschi I, Alfieri O. Over 15 years: the advancement of transcatheter mitral valve repair. Ann Cardiothorac Surg 2021;10(1):15-27. doi: 10.21037/ acs-2020-mv-18 procedure for which patient? EuroIntervention 2019;15:867-74.

51. Feldman T, Fernandes E, Levisay JP. Transcatheter mitral valve repair/replacement for primary mitral regurgitation. Ann Cardiothorac Surg 2018;7:755-63. 